\title{
ACTIVATED BICARBONATE SOLUTIONS AS MODELS OF CONFINED ONTIC OPEN SYSTEM AND PROTOTYPES OF LIVING RESPIRING SYSTEMS
}

\author{
V.L. VOEIKOV, DO MING HA, O.G. MUKHITOVA, N.D. VILENSKAYA, \\ S.I. MALISHENKO \& A.S. BOGACHUK \\ Faculty of Biology, Lomonosov Moscow State University, Moscow, Russia.
}

\begin{abstract}
Addition of $\mathrm{H}_{2} \mathrm{O}_{2}$ in sub-millimolar concentrations to $1-5 \mathrm{mM} \mathrm{Na} / \mathrm{K}$-bicarbonate solutions initiates in them a process accompanied with low-level photon emission amplified with luminol. Photon emission lasts without decay for many months in samples isolated from air and ambient light. Photon emission intensity reveals monthly and circadian rhythms. Amplitude of photon emission intensity from test-tubes filled with active solutions increases two- to threefold on periods coinciding with the eclipses of the moon and the sun. Preparations of water-soluble fullerenes in dilutions equivalent to their concentrations $10^{-13}, 10^{-15}$ and $10^{-19}-10^{-20} \mathrm{M}$ increased photon emission two- to threefold while intermediate dilutions affected it much weaker. We suggest that addition of $\mathrm{H}_{2} \mathrm{O}_{2}$ to aqueous bicarbonate solutions initiates in them cyclic chain reactions in which water is oxidized with oxygen. Bicarbonate may stabilize these cyclic reactions due to its ability to participate in free radical reactions. Extremely long-lasting photon emission (high density electromagnetic energy) from activated bicarbonate solutions indicates that they reside in stable non-equilibrium (excited) state supported presumably by continuous efflux of low density (electromagnetic) energy from the environment. Such systems may represent a model of Confined Ontic Open Systems able to transform low grade energy into high grade energy. It is notable that bicarbonate is the necessary constituent of cytoplasm of aerobic cells and of important biological liquids, in particular of blood plasma. Normal and healing drinking waters also usually represent bicarbonate solutions.

Keywords: bicarbonate, hydrogen peroxide, non-equilibrium state, water, photon emission.
\end{abstract}

\section{INTRODUCTION}

Previously we found that addition of $\mathrm{Fe}$ (II) salts $\left(\mathrm{FeSO}_{4}\right.$ or $\left.\mathrm{FeCl}_{2}\right)$ in the range of concentrations of $10-50 \mathrm{mkM}$ to bicarbonate artesian waters or aqueous bicarbonate solutions induces a wave of photon emission (PE) from them. Intensity of the wave was boosted in the presence of luminol, the probe for the reactive oxygen species (ROS). PE-waves in bicarbonate aqueous solutions could be induced in them by addition of $\mathrm{Fe}$ (II) in the presence of luminol many weeks after their preparation provided that they were in contact with air [1]. Induction by Fe(II) of luminol-amplified PE indicated that spontaneous chain reactions with ROS participation continuously flow in aqueous bicarbonate solutions. However, amplitudes of PE-waves decreased significantly if a bicarbonate solution was kept in a sealed vessel. After its opening amplitude of PE-wave increased again indicating that contact with the air was needed for the persistence of processes responsible for PE.

Here we report a peculiar process accompanied with $\mathrm{PE}$ that is initiated by the addition of $\mathrm{H}_{2} \mathrm{O}_{2}$ to aqueous bicarbonate solutions. The process may proceed in hermetically closed test-tubes and in darkness for many months without decay. The process exhibits extremely high sensitivity to the action of physical and chemical factors of ultra-weak intensity. Aqueous bicarbonate solution in which such process is induced may represent a model of Confined Ontic Open Systems as described in [2]. This aqueous system is able to transform low grade energy incoming from the environment into free energy of photon emission and in high grade energy of its stable non-equilibrium state [3] sensitive to ultra-weak environmental factors. It is notable that bicarbonate is the necessary constituent of cytoplasm of aerobic cells and of biological liquids, in particular of blood plasma. Normal and healing drinking waters are also usually bicarbonate solutions. Steady activity of bicarbonate aqueous 
solutions induced by rather weak factors may represent a property important for many vital functions of individual living systems as well as for the natural environmental processes.

\section{EXPERIMENTAL}

Solutions of $\mathrm{Na} / \mathrm{KHCO}_{3}(5 \mathrm{mM}, \mathrm{Na}: \mathrm{K}=3: 1)$ were prepared using bi-distilled deionized water $\mathrm{H}_{2} \mathrm{O}_{2}$ taken from 3\% stock solution was introduced to bicarbonate solutions to final concentrations of $0,01 \%$. Luminol taken from $10 \mathrm{mM}$ stock solution in DMSO was introduced in bicarbonate solutions to the final concentration of $25 \mathrm{mkM}$. Bicarbonate solution 1-ml aliquots were transferred to $1.5 \mathrm{ml}$ Eppendorf test-tubes that were tightly closed with caps and kept in dark boxes at room temperature $\left(22-24^{\circ} \mathrm{C}\right)$. In experiments where effects of hydrated fullerenes upon PE were studied serial dilutions of hydrated fullerenes C60 from 1-mM stock solution of fullerenes in 5-mM bicarbonate solution obtained from G.V. Andrievsky [4] were prepared to final concentrations from $1 \times 10^{-9}$ to $1 \times$ $10^{-20} \mathrm{M} . \mathrm{H}_{2} \mathrm{O}_{2}$ and luminol were added to 1-ml portions of these solutions in Eppendorf test-tubes to initiate the process accompanied with PE.

$\mathrm{PE}$ was registered using single photon counters Biotox 7 and Biotox 7A. The first one is equipped with photomultiplier FEU-101 with the photocathode being $1 \mathrm{~cm}$ in diameter (dark current $\sim 10$ counts/sec). Sample chamber of this detector may be thermostated with the precision of $\pm 0.2^{\circ} \mathrm{C}$. Biotox $7 \mathrm{~A}$ is equipped with photomultiplier $9750 \mathrm{QB} / 1$ (EMI Electronics) with the photocathode being $5 \mathrm{~cm}$ in diameter (dark current $\sim 25$ counts $/ \mathrm{sec}$ ). Both photomultipliers are sensitive to photons in the range of $\lambda=400-500 \mathrm{~nm}$ with the maximum sensitivity of $\lambda=450 \mathrm{~nm}$. Biotox 7 was used for continuous recording of $\mathrm{PE}$ from a single sample for days and weeks without interruption, and Biotox 7A was used for daily counts of the series of samples.

\section{RESULTS}

Figure 1 demonstrates typical dynamics of $\mathrm{PE}$ from sealed test tubes containing $1 \mathrm{ml}$ of bicarbonate solutions $(5 \mathrm{mM})$ into which $\mathrm{H}_{2} \mathrm{O}_{2}$ and luminol were introduced on 2 July 2008. Regular measurements of PE were performed initially several times a week and later each week at one and the same time of the day. It can be seen that during several months of monitoring of PE from the solutions average PE intensity did not change significantly, though some trend for its decline may be observed. Still, on 15 June 2009 (the last point at the plot), intensity of photon emission exceeded background count rate of the photomultiplier (counts without the sample) 160 -fold. We also observed that after addition of a small aliquot (e.g. $25 \mathrm{mkl}$ ) of non-activated $5 \mathrm{mM}$ bicarbonate solution to $1 \mathrm{ml}$ of an active $5 \mathrm{mM}$ bicarbonate solution PE intensity immediately increases many-fold and relaxes to the initial level for several hours (data not shown). This indicates that the system resides in a highly nonequilibrium state and may release excess of energy in response to even slight 'irritation'. Monthly rhythms in variations of PE intensity can be seen in Fig. 1. This regularity did not correlate with the variations of ambient temperature in the room where measurements were performed that varied in the range $22-24^{\circ} \mathrm{C}$.

To study the regularities of variability of PE intensity in more detail, single samples with activated bicarbonate solution were fixed in the thermostatic sample compartment of the Biotox 7 photon detector and continuous recording of PE was performed for several weeks without interference into the process. A representative week-long recording of PE intensity from a sample with $5 \mathrm{mM}$ bicarbonate solution where the reaction was initiated on 10 March 2009 is presented in Fig. 2. Circadian rhythm of variations of $\mathrm{PE}$ with the minimum of $\mathrm{PE}$ around 7-9 h AM and maximum around 18-20 h PM can be seen in Fig. 2, though daily patterns of variations of PE could differ on different days. It should be stressed that during the whole period of monitoring the sample fixed in the thermostatic chamber was completely isolated from ambient light. 


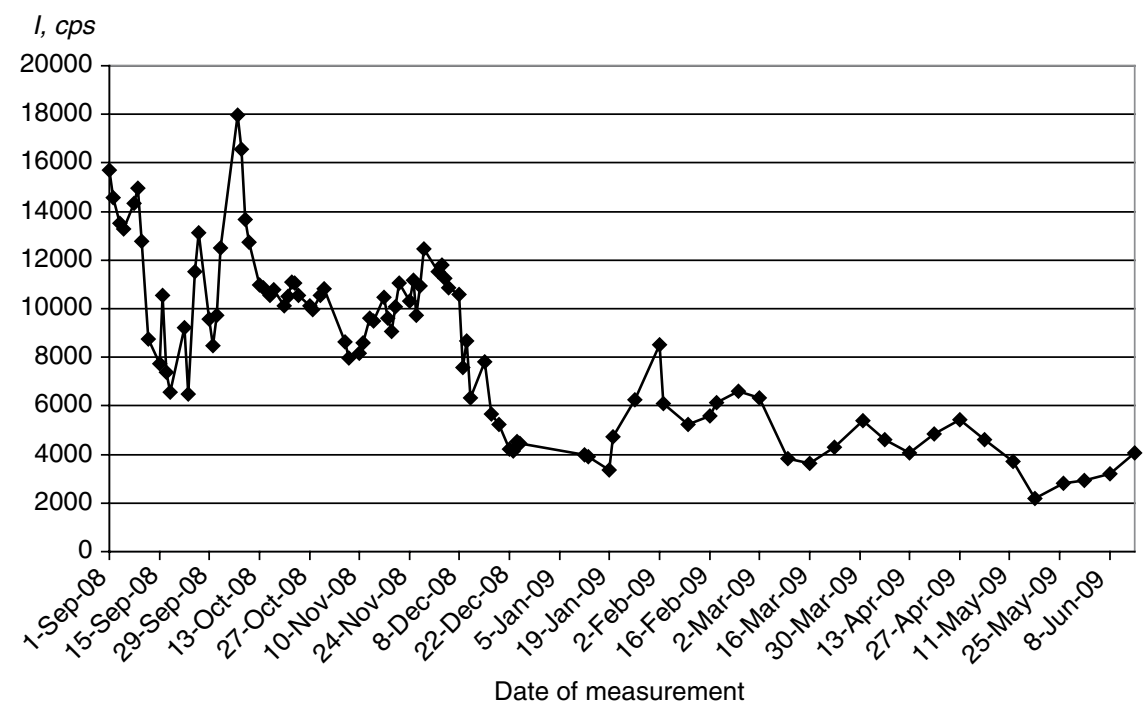

Figure 1: Luminol-amplified PE from 1-ml samples of bicarbonate solutions (5 mM) to which $\mathrm{H}_{2} \mathrm{O}_{2}$ ( $0.01 \%$ final concentration) and luminol ( $25 \mathrm{mkM}$ final concentration) was added on 2 July 2008 , after which test tubes were tightly sealed and kept in a dark box before the measurements. The curve represents the results of consecutive measurements of PE made four-five times a week from 1 September 2008 to the end of December 2008 and once a week from January to June 15,2009 . The points represent mean values of intensity from triplicate samples. SD of the mean values were in the range $5-15 \%$ on different days of measurement.

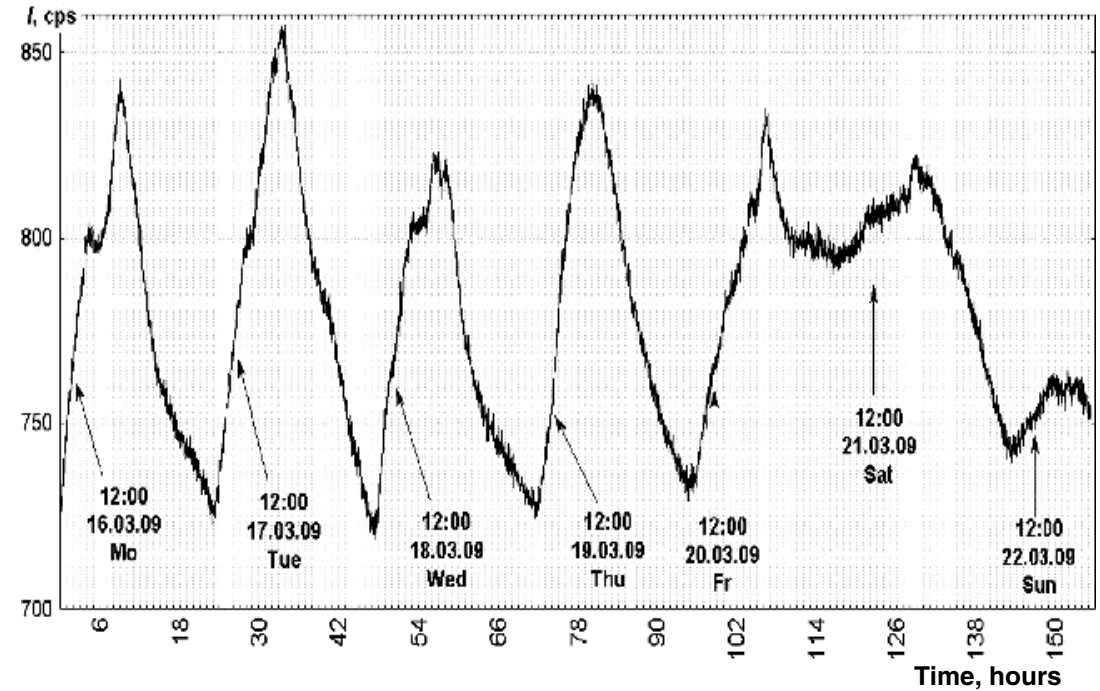

Figure 2: Monitoring of PE from a sample with 5-mM bicarbonate solution where the process was initiated by the addition of $\mathrm{H}_{2} \mathrm{O}_{2}$ and which was installed in the photon counter on 10 March 2008. Record of data presented here starts at 9:20 AM on 16 March. Abscissa time (hours) after the start of the record. Data was obtained with $1 \mathrm{sec}$ sampling time; the data presented here is moving average aggregation of consecutive 120 time points. 
The chamber represents the metal case located in a metal body of the photon detector. Dark current of the counter did not reveal any significant circadian oscillations. When PE from two different samples was recorded in parallel using two different photon counters for more than 2 days patterns of PE intensity variations in these samples were similar. Thus, one may suggest that the general pattern of PE changes and its peculiarities represent the reaction of the process going on in activated bicarbonate solution upon circadian oscillations of certain weak environmental physical factors presumably of electromagnetic nature.

More or less regular circadian rhythms of PE intensity that were generally observed during longterm monitoring of PE from single samples were drastically disturbed during the week that started on 9 February 2009. On this day the full moon eclipse took place in Moscow. It started on 9 February at 17:34 h and exactly at this moment of time PE intensity started to increase dramatically (Fig. 3). The moon's eclipse was full at 19:38 and a spike on the kinetic curve was observed just at this moment. After the moon's eclipse ended PE intensity did not decrease to its initial values, but started to vary with a circadian pattern at the level exceeding the previous one by two- to threefold. Surprisingly $47 \mathrm{~h}$ after the start of the moon's eclipse photon emission rapidly declined to the level preceding the eclipse. PE intensity again rose more than twofold 3 days after the moon's eclipse and $2 \mathrm{~h}$ later it fell to the initial level. And again a spike appeared on the descending slope of the curve exactly $72 \mathrm{~h}$ ( 3 days) after the full eclipse moment.

Exact correlations between PE and time schedule of the moon's eclipse can hardly be explained by a mere coincidence though currently it is difficult to suggest any hypothesis on the mechanism of action of the moon's eclipse on the activated bicarbonate solutions.

We also studied the effect of preparations of hydrated fullerenes $\mathrm{C} 60(\mathrm{HyFn})$ in low and ultra-low doses on PE intensity from activated bicarbonate solutions. Hydrated fullerenes (HyFn) is an aqueous solution of fullerene molecules $\mathrm{C60}$, prepared in such a way that each fullerene molecule is

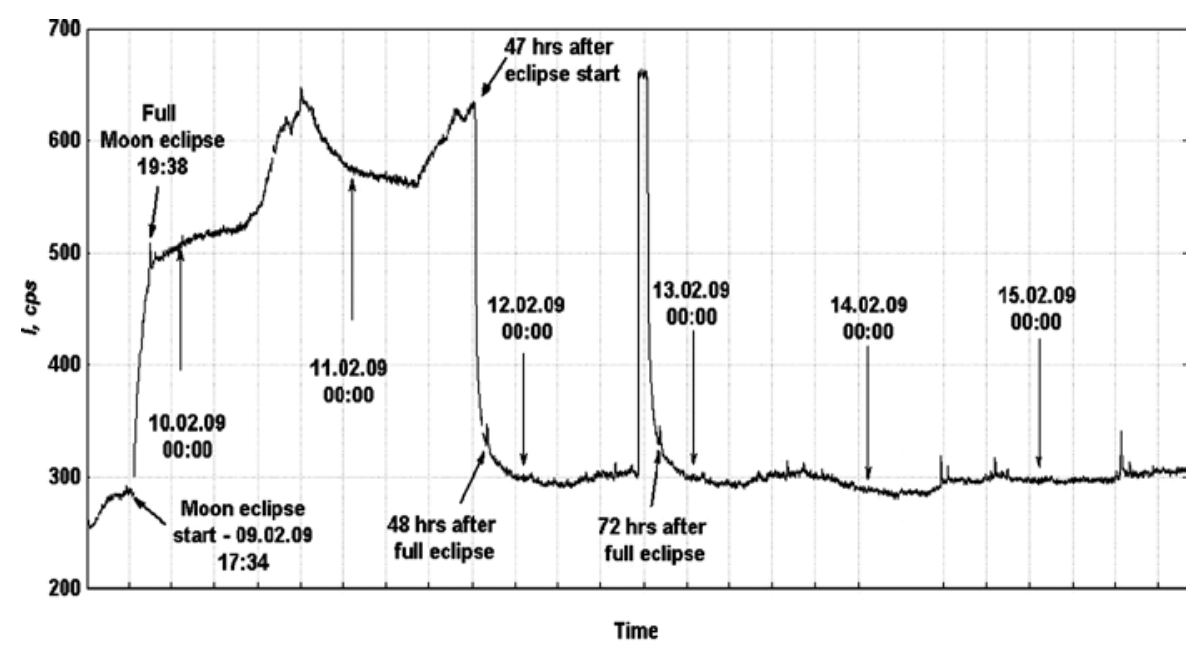

Figure 3: Monitoring of luminol-enhanced PE from a sample with $\mathrm{H}_{2} \mathrm{O}_{2}$ activated $5 \mathrm{mM}$ bicarbonate solution prepared on 11 November 2008. The continuous session of PE monitoring has been started on 26 January 2009. The record presented here started at 11:40 AM on 9 February 2009. Original data was collected with $1 \mathrm{sec}$ sampling time; the data presented are moving average aggregation of consecutive 120 time points. Thick arrows mark spikes in PE intensity coinciding with the special time points. 
enclosed in a multilayer aqueous shell [5]. HyFn display a wide spectrum of biological action both in vivo and in vitro. HyFn given to animals in low doses efficiently protects them from oxidative stress, and formally behave as strong antioxidants [6]. On the other hand HyFn have reducing properties and may serve as electron donors for the dissolved oxygen, exhibiting pro-oxidant activity. Taking into consideration that fullerenes C60 are themselves chemically inert and these molecules are covered with multilayered water shells in HyFn preparations one may suggest that the whole spectrum of action of HyFn results from specific properties of water surrounding them [7].

Decimal dilutions of HyFn were made in $5 \mathrm{mM}$ bicarbonate solutions to make their final concentrations from $10^{-11}$ to $10^{-20}$. After addition of $\mathrm{H}_{2} \mathrm{O}_{2}$ initial PE intensity in all the samples with HyFn was higher than in the control, but on the next day a non-monotonic polymodal dose response was observed. This peculiar dose-dependence of PE intensity from bicarbonate solutions with serial HyFn dilutions measured on day 5 after their activation is illustrated in Fig. 4. PE intensity in the samples containing HyFn in doses equivalent to $10^{-13}, 10^{-15}-10^{-16}$ and $10^{-19}-10^{-20} \mathrm{M}$ was, respectively, two-, five-, threefold, and more than twofold higher than in control samples, while intermediate doses stimulated PE intensity less significantly. Non-monotonous dose dependence is characteristic of the effects of ultra-low doses of biologically active substances on biological testsystems in vivo and in vitro [8,9], but such dependence of their effect on an aqueous system with a 'simple' chemical composition is demonstrated to our knowledge for the first time.

\section{DISCUSSION}

It is well known that in chemical reactions in which free radicals participate energy of electronic excitation equivalent to the energy of photons of visible and UV-range of the spectrum is generated. When appropriate luminescent (fluorescent) or chemiluminescent probes are added to reaction systems where free radical recombination and decomposition of highly unstable intermediate peroxy

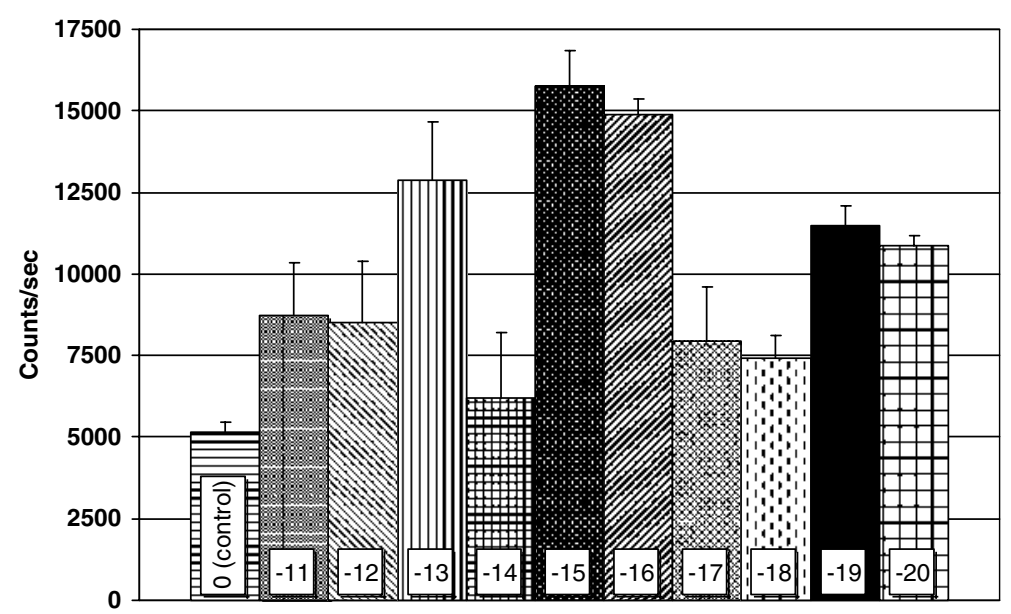

[Hydr. Fullerenes], Ig (M)

Figure 4: Dose-dependence of PE intensity from $5 \mathrm{mM}$ bicarbonate solutions containing serial HyFn dilutions equivalent to concentrations of $\mathrm{HyFn}$ indicated on the respective bars before their activation with $\mathrm{H}_{2} \mathrm{O}_{2}$. Each bar represents a mean value of $\mathrm{PE}$ intensity from triplicate samples ( \pm SD of the mean) measured on day 5 after activation and sealing test tubes with the solutions. 
compounds take place photon emission may be registered using sensitive photon detectors. However, in the vast majority of such systems PE usually sooner or later decays due to the consumption of reagents. Bicarbonate aqueous solutions to which $\mathrm{H}_{2} \mathrm{O}_{2}$ in rather low concentration was added (and that should rapidly decompose, see later) seem to be unusual in this respect and demonstrate the whole set of peculiar properties. $\mathrm{H}_{2} \mathrm{O}_{2}$ initiates in bicarbonate solutions the process accompanied with PE that does not decay for many months in the complete darkness and when no exchange of matter (oxygen and other gases) with the environment takes place. This means that the system has the sustained ability to generate energy of electronic excitation without 'food' and access to high density energy that could drive photosynthetic-like reactions. Besides the system is able to accumulate high density energy as it can react to subtle irritations by strong and prolonged rising up of PE intensity. Indeed, PE intensity from activated bicarbonate solutions as reported here is rather low, but according to our preliminary data it can be increased more than 100-fold by the addition of small quantities of peroxidase and sustain at this increased level for many days without fading out. It is premature to suggest any more or less detailed mechanism of processes that are responsible for permanently excited state of activated bicarbonate solution. However, some preconditions for such a model can be discussed.

Liquid water as it follows from the Quantum Field Theory (QFT) approach may be regarded as a bi-phasic system: a phase represented by coherent domains (CDs) interspersed in non-coherent water behaving as a dense gas. A coherent domain is a reservoir of quasi-free electrons, and it has reducing properties, able to donate electron to the appropriate oxidizer [10]. According to this theory CDs are stabilized at the interfaces between the liquid and the hydrophilic surface since the attraction between the liquid and the surface could be strong enough to compensate for the disruptive effect of thermal collisions. Lifetime of CDs could thus be days, weeks or even months. Interfacial water permanently exhibits a coherent structure, whose long range dynamics become observable [11]. In fact, in a series of recent experimental papers from the laboratory of G.H. Pollack it has been proved that a thick layer of water is built up near different hydrophilic surfaces including water-air interface with liquid crystalline properties and high reducing potential [12].

According to the concept developed earlier by one of us (V.L.V.), reduction of oxygen present in liquid water by water (looking from another perspective - water oxidation with oxygen) is not an improbable process, and there are many experimental facts indicating that under certain conditions it readily goes on [13]. Direct water oxidation is a kind of 'water burning'. Such a reaction is a cyclic one because its products are again water and oxygen. 'Water burning', as any other burning is accompanied with generation of energy of electronic excitation. QFT of water according to which CDs represent an electron donor phase and the other phase represent less organized water and contains oxygen - provide theoretical support for this concept. On the other hand, Pollack's data demonstrate that stable charge separation takes place in multiple aqueous systems under 'normal' conditions; in principle it may serve a source of free energy of electron transfer from liquid crystalline water to electron acceptors in bulk water. However, the direct water oxidation with oxygen needs either the continuous supply of energy of activation or catalysis allowing to decrease the energy barrier for 'water burning' to proceed.

Carbonates present in water may play the role of such versatile catalysts. On the one hand they support water structuring [14], increasing the potential difference between the two phases, and on the other catalyze the process of water 'burning' due to their ability to participate in free radical reactions. Thus, if the appropriate electron donors are available one-electron reduction of $\mathrm{CO}_{2}$ to carbon dioxide anion radical $\left(\mathrm{CO}_{2}^{-*}\right)$ is thermodynamically favorable [15]. This radical is a strong reducer, and it can reduce oxygen, serving as a kind of a shuttle between the phase of reducing water and oxygen. On the other hand in the course of water oxidation intermediate products arise, and one of 
them, hydroxyl radical $\left(\mathrm{HO}^{\circ}\right)$, reacts with bicarbonate anions that turn into carbonate anion radical $\left(\mathrm{CO}_{3}^{-}\right)$. The latter may support water oxidation, oxidize hydrogen peroxide [16], give rise to the emergence of organic compounds, such as oxocarbons [17]; the latter are able to originate cyclic red/ ox reactions. A network of coupled and mutually supporting red/ox reactions emerges yielding energy of electronic excitation. Thus, it may start only when oxygen concentration exceeds a certain threshold, and only if it is initiated with a triggering stimulus (a 'spark') of high enough potential.

Regarding the role of $\mathrm{H}_{2} \mathrm{O}_{2}$ it should be stressed that combustion generally proceeds as the branching chain process [18]. Thus, it may start only when oxygen concentration exceeds a certain threshold, and only if it is initiated with a triggering stimulus (a 'spark') of high enough potential. $\mathrm{H}_{2} \mathrm{O}_{2}$ introduced into a bicarbonate solution more or less rapidly decomposes, in particular due to reaction with bicarbonate [19]. On the one hand $\mathrm{H}_{2} \mathrm{O}_{2}$ decomposition provides a 'spark' - a triggering impulse for the reaction initiation and on the other oxygen concentration in the system increases allowing overcoming the threshold needed for the kindling of chain reactions. After burning initiation energy released in its course promotes both fuel and oxygen excitation, resulting in the reinforcement of the burning process.

Indeed this is a very preliminary sketch of the network of physical-chemical processes that develop in bicarbonate solutions activated with hydrogen peroxide, but whatever the mechanism providing for the stable non-equilibrium state of bicarbonate aqueous systems, its capability for permanent photon emission demands sustained energy supply. The only source of it under the given conditions is a thermal (or low density electromagnetic energy) bath in which the system resides. As the structural temperature of organized water is lower than that of less organized water with which it is in contact, a temperature (or radiation) gradient between these two water phases exists. So organized water can constantly draw heat (or low density electromagnetic energy) from the environment and transform it into energy of much higher density - energy of electronic excitation. From this it follows that bicarbonate solutions represent step-up energy transformers and the law of energy conservation is not violated at all. These systems are confined in a sense that they are closed for matter exchange with the environment. On the other hand these are open systems as they can draw low grade energy, convert it into much higher grade energy of their excited state and release excess of it in the form of high grade free energy of photon emission. Besides they are open to ultra-weak 'irritations' coming from their environment that may be looked upon as their openness to information. Thus activated bicarbonate aqueous solutions represent in our opinion an important example of Confined Ontic Open Systems as their properties agree with many features of such systems as defined by E. Tiezzi et al. [2]. These systems may be considered 'Ontic' because essentially they represent a simple model of all biological liquids and many natural waters.

A significant part of water in living matter has the properties of a liquid crystalline phase. On the other hand, carbonates are the necessary constituent of living systems' water. In this respect it is interesting to note the key role of carbonates and in particular bicarbonates in aerobic respiration was discovered already in nineteenth century [20]. According to renowned physiologist Yandell Henderson decrease of carbonates levels in the organism below some critical level especially in brain may results in fatigue and death due to respiration cessation [21]. Henderson considered that the effects of carbonates are mediated by regulation of acid-base balance by them, but made a reservation that carbonates may exert some more specific action upon molecular targets. Bicarbonates are the necessary constituents of culture medium for normal proliferation of cultured cells in vitro [22]. They also turned out to modulate oxidation, peroxidation and nitration both in vivo, and in vitro due to their striking effects on the activity of the enzymes involved in metabolism of ROS [23]. It cannot be excluded that the role carbonates play in respiration may be at least partially related to phenomena described earlier. 
According to the concept presented here the suggested oxidation-reduction cycle of water ( water burning' = 'water respiration') catalyzed by carbonates is likely to be the fundamental source of energy for living systems, the basis of their residence in a highly excited state, their sensitivity to resonant external 'irritants' of even very low intensity. On the whole it is interesting to speculate that intrinsic sustained activity of water containing carbonates is likely to be the necessary and sufficient condition for emergence and existence of living systems.

\section{REFERENCES}

[1] Voeikov, V., Asfaramov, R., Koldunov, V., Kononov, D., Novikov, C. \& Vilenskaya, N., Chemiluminescent analysis reveals spontaneous oxygen-dependent accumulation of high density energy in natural waters. Clinical Laboratory, 49, pp. 569, 2003.

[2] Tiezzi E., Cecconi, G. \& Marchettini N., Confined ontic open systems. International Journal of Design \& Nature and Ecodynamics, 5(1), pp. 3-9, 2010.

[3] Voeikov, V.L. \& Del Giudice, E., Water respiration - The basis of the living state. Water: A Multidisciplinary Reserch Journal, 1, pp. 52-75, 2009. http://waterjournal.org/content/ view/45/64/

[4] Andrievsky, G.V., Klochkov, V.K., Bordyuh, A. \& Dovbeshko, G.I., Comparative analysis of two aqueous-colloidal solutions of c60 fullerene with help of FT-IR reflectance and UVVIS spectroscopy. Chemical Physical Letters, 364, pp. 8-17, 2002. doi:10.1016/S0009-2614(02)01305-2

[5] Andrievsky, G.V., Kosevich, M.V., Vovk, O.M., Shelkovsky, V.S. \& Vashchenko, L.A., On the production of an aqueous colloidal solution of fullerenes. Journal of the Chemical Society, Chemical Communication, 12, pp. 1281, 1995. doi:10.1039/c39950001281

[6] Tikhomirov, A.A., Nedzvetskii, V.S., Lipka, M.V., Andrievskii, G.V. \& Klochkov, V.K., Chronic alcoholization-induced damage to astroglia and intensification of lipid peroxidation in the rat brain: protector effect of hydrated form of fullerene pp. 60. Neurophysiology, 39(2), 2007. doi:10.1007/s11062-007-0015-8

[7] Voeikov, V.L., Do Ming Ha, Vilenskaya, N.D., Malishenko, S.I. \& Andrievskii, G.V., Effects of hydrated fullerenes in sub-nanomolar concentrations upon redox reactions in aqueous systems and on enzyme activity. Proceedings of the XII International Congress on Bioelectrography, St. Peterburg, pp. 95-96, 2008.

[8] Davenas, E., Bauvais, F., Amara, J., Oberbaum, M., Robinson, B., Miadonna, A., Tedeschi, A., Pomeranz, B., Forner, P., Belon, P., Sainte-Laudi, J., Poitevin, B. \& Benveniste J. Human, Basophil degranulation triggered by very diluted antiserum against Ig E. Nature, 333, pp. 267-272, 1988. doi:10.1038/333816a0

[9] Maltseva, E.L., Palmina, N.P. \& Burlakova, E.B., Natural (alpha-tocopherol) and synthetic (phenosan potassium salt) antioxidants regulate the protein kinase $\mathrm{C}$ activity in a broad concentration range $\left(10^{-4}-10^{-20} \mathrm{M}\right)$. Membrane and Cell Biology, 12, pp. 251-268, 1998.

[10] Arani, R., Bono, I., Del Giudice, E. \& Preparata, G., QED Coherence and the Thermodynamics of water. International Journal of Modern Physics B, 9, pp. 1813-1841, 1995. doi:10.1142/ S0217979295000744

[11] Del Giudice, E. \& Tedeschi, A., Water and the autocatalysis in living matter. Electromagnetic Biology and Medicine, 28, pp. 46, 2009. doi:10.1080/15368370802708728

[12] Pollack, G.H., Water, energy and life: fresh views from the water's edge. International Journal of Design \& Nature and Ecodynamics, 5(1), pp. 27-29, 2010.

[13] Voeikov, V.L., Biological oxidation: over a century of hardship for the concept of active oxygen. Cellular and molecular biology (Noisy-le-Grand, France), 51, pp. 663-675, 2005. 
[14] Pauling, L., A molecular theory of general anesthesia. Science, 134, pp. 15-21, 1961. doi:10.1126/science.134.3471.15

[15] Halmann, M.M., Chemical fixation of carbon dioxide, CRC Press, pp. 11-15, 1993. Tanaka, T., Molecular orbital studies of the two-electron reduction of carbon dioxide to give formate anion, Memories Fukui Univ. Technol., 28, Pt.1, pp. 223-230, 1993.

[16] Komissarov, G.G., Photosynthesis: Physical-chemical Approach. Moscow, pp. 154-170, 2003.

[17] Mazellier, P., Transformation of carbendazim induced by the $\mathrm{H}_{2} \mathrm{O}_{2} / \mathrm{UV}$ system in the presence of hydrogenocarbonate ions: involvement of the carbonate radical. New Journal of Chemistry, 26, pp. 1784-1790, 2002. doi:10.1039/b204332g

[18] Voeikov, V.L. \& Naletov, V.I., Weak photon emission of non-linear chemical reactions of amino acids and sugars in aqueous solutions. Biophotons, eds. J-J Chang, J. Fisch, F-A. Popp, Kluwer Academic Publishers: Dortrecht, pp. 93-108, 1998.

[19] Richardson, D.E., Yao, H., Frank, K.M. \& Bennett, D.A., Equilibria, kinetics, and mechanism in the bicarbonate activation of hydrogen peroxide: oxidation of sulfides by peroxymonocarbonate. Journal of the American Chemical Society, 122, pp. 1729-1739, 2000. doi:10.1021/ ja9927467

[20] Miescher, F. Bemerkungen, zur Lehre von den Athembewegungen. Archives of Anatomy and Physiology, Physiol Abth, pp. 3555-3561, 1885.

[21] Henderson, Y., Adventures in respiration. Modes of Asphyxiation and Methods of Resuscitation, Williams \& Wilkins: Baltimore, 1938.

[22] Mitaka, T., Sattler, G.L. \& Pitot, H.C., The bicarbonate ion is essential for efficient dna synthesis by primary cultured rat hepatocytes. In Vitro Cellular and Developmental Biology, 27A, pp. 549-556, 1991. doi:10.1007/BF02631285

[23] Medinas, D.B., Cerchiaro, G., Trindade, D.F. \& Ohara A., The carbonate radical and related oxidants derived from bicarbonate buffer. IUBMP Life, 59, pp. 255-262, 2007. doi:10.1080/15216540701230511 